\title{
THREE-FORMS AND ALMOST COMPLEX STRUCTURES ON SIX-DIMENSIONAL MANIFOLDS
}

\author{
MARTIN PANÁK ${ }^{\bowtie}$ and JIŘÍ VANŽURA \\ (Received 23 July 2003; revised 10 August 2006) \\ Communicated by C. D. Hodgson
}

\begin{abstract}
This paper deals with 3-forms on six-dimensional manifolds, the first dimension where the classification of 3-forms is not trivial. It includes three classes of multisymplectic 3-forms. We study the class which is closely related to almost complex structures.
\end{abstract}

1991 Mathematics subject classification: 53C15, 58A10.

Keywords and phrases: 3 -form, almost complex structure, six-dimensional manifold.

\section{Introduction}

There is growing interest in the study of 3-forms among geometers and physicists in the recent years. There are various geometrical structures connected with different types of 3-forms on manifolds.

The connections with totally skew-symmetric torsion, which is a 3-form, play an important role in the research of Thomas Friedrich (see, for example, $[\mathrm{F}]$ ).

Nigel Hitchin and his school also show an interest in 3-forms $[\mathrm{H}, \mathrm{W}]$. There are three orbits of the action of the group GL $(6, \mathbb{R})$ on the multisymplectic (full-rank) 3 -forms on a six-dimensional vector space. There is either a tangent, complex, or product structure connected with a 3-form on a six-dimensional vector space. The kind of structure depends on which of the three orbits the form belongs to. We speak about the forms of product type, of complex type or of tangent type accordingly. The notion of a 3-form of the given type on the manifold can be defined in the obvious way. We study closely the 3 -forms of complex type and we construct the associated complex structure in a different (and we think simpler) way than Hitchin in $[\mathrm{H}]$. Furthermore, we investigate the interplay between the integrability of the complex

The first author was supported by the Grant Agency of the Czech Republic, grant No. 201/02/1390. The second author was supported by the Grant Agency of Czech Academy of Sciences, grant no. A 1019204.

(C) 2008 Australian Mathematical Society 1446-7887/08 \$A2.00+0.00 
structure associated with a given 3-form of complex type and the existence of the linear symmetric connection, which preserves the form. The result is stated in Theorem 13, which can be regarded as the Darboux theorem for the 3-forms of complex type.

THEOREM. Let $\omega$ be a real 3-form of complex type on a six-dimensional differentiable manifold $M$. Let $J$ be the almost complex structure on $M$ such that, for any vector fields $X_{1}, X_{2}, X_{3} \in \mathfrak{X}(M)$,

$$
\omega\left(J X_{1}, X_{2}, X_{3}\right)=\omega\left(X_{1}, J X_{2}, X_{3}\right)=\omega\left(X_{1}, X_{2}, J X_{3}\right) .
$$

Then there exists a symmetric connection $\tilde{\nabla}$ on $M$ such that $\tilde{\nabla} \omega=0$ if and only if the following conditions are satisfied:

(i) $d \omega=0$;

(ii) the almost complex structure $J$ is integrable.

\section{The orbits of the 3-forms on six-dimensional spaces}

Let $V$ be a real vector space. Recall that a $k$-form $\omega(k \geq 2)$ is said to be multisymplectic if the homomorphism

$$
\iota: V \rightarrow \Lambda^{k-1} V^{*}, \quad v \mapsto \iota_{v} \omega=\omega(v, \ldots),
$$

is injective. There is a natural action of the general linear group $\operatorname{GL}(V)$ on $\Lambda^{k} V^{*}$, and also on $\Lambda_{m s}^{k} V^{*}$, the subset of the multisymplectic forms. Two multisymplectic forms are called equivalent if they belong to the same orbit of the action. For any form $\omega \in \Lambda^{k} V^{*}$ we define a subset

$$
\Delta(\omega)=\left\{v \in V:\left(\iota_{v} \omega\right) \wedge\left(\iota_{v} \omega\right)=0\right\} .
$$

If $\operatorname{dim} V=6$ and $k=3$, the subset $\Lambda_{m s}^{3} V^{*}$ consists of three orbits. Let $e_{1}, \ldots, e_{6}$ be a basis of $V$ and $\alpha_{1}, \ldots, \alpha_{6}$ the corresponding dual basis. Representatives of the three orbits can be expressed in the form:

(1) $\omega_{1}=\alpha_{1} \wedge \alpha_{2} \wedge \alpha_{3}+\alpha_{4} \wedge \alpha_{5} \wedge \alpha_{6}$;

(2) $\omega_{2}=\alpha_{1} \wedge \alpha_{2} \wedge \alpha_{3}+\alpha_{1} \wedge \alpha_{4} \wedge \alpha_{5}+\alpha_{2} \wedge \alpha_{4} \wedge \alpha_{6}-\alpha_{3} \wedge \alpha_{5} \wedge \alpha_{6}$;

(3) $\omega_{3}=\alpha_{1} \wedge \alpha_{4} \wedge \alpha_{5}+\alpha_{2} \wedge \alpha_{4} \wedge \alpha_{6}+\alpha_{3} \wedge \alpha_{5} \wedge \alpha_{6}$.

We speak of multisymplectic forms of product type (first form), or of complex type (second form), or of tangent type (third form) depending on which orbit they belong to. The orbits can be characterized as follows:

(1) $\omega$ is of product type if and only if $\Delta(\omega)=V^{a} \cup V^{b}$, where $V^{a}$ and $V^{b}$ are threedimensional subspaces satisfying $V^{a} \cap V^{b}=\{0\}$;

(2) $\omega$ is of complex type if and only if $\Delta(\omega)=\{0\}$;

(3) $\omega$ is of tangent type if and only if $\Delta(\omega)$ is a three-dimensional subspace.

The forms $\omega_{1}$ and $\omega_{2}$ have equivalent complexifications. From this point of view the forms of tangent type are exceptional. See [V] for further details.

A multisymplectic $k$-form on a manifold $M$ is a section of $\Lambda^{k} T^{*} M$ such that its restriction to the tangent space $T_{x} M$ is multisymplectic for any $x \in M$, and is of type 
$i$ in $x \in M, i=1,2,3$, if the restriction to $T_{x} M$ is of type $i$. A multisymplectic form on $M$ can change its type as can be seen from the the following example:

$$
\begin{aligned}
\sigma= & d x_{1} \wedge d x_{2} \wedge d x_{3}+d x_{1} \wedge d x_{4} \wedge d x_{5}+d x_{2} \wedge d x_{4} \wedge d x_{6} \\
& +\sin \left(x_{3}+x_{4}\right) d x_{3} \wedge d x_{5} \wedge d x_{6}+\sin \left(x_{3}+x_{4}\right) d x_{4} \wedge d x_{5} \wedge d x_{6}
\end{aligned}
$$

a 3 -form on $\mathbb{R}^{6}$. Then $\sigma$ is of type 3 on the submanifold given by the equation $x_{3}+x_{4}=k \pi, k \in \mathbb{N}$. If $x_{3}+x_{4} \in(k \pi,(k+1) \pi), k$ even, then $\sigma$ is of type 1 and if $x_{3}+x_{4} \in(k \pi,(k+1) \pi), k$ odd, then $\sigma$ is of type 2 . We point out that $\sigma$ is closed and invariant under the action of the group $(2 \pi \mathbb{Z})^{6}$ and we can factor $\sigma$ to get a form changing the type on $\mathbb{R}^{6} /(2 \pi \mathbb{Z})^{6}$, which is the six-dimensional torus, that is, $\sigma$ is closed on a compact manifold. The goal of this paper is to study the forms of complex type. We denote $\omega=\omega_{2}$.

\section{Three-forms of complex type on vector spaces}

In this section, in Proposition 7, we associate a 3-form of complex type on a six-dimensional vector space $V$ with the complex structure on the vector space (thereby justifying the name). In Proposition 8 we associate the couple (a 3-form and the corresponding complex structure) with the unique complex 3-form on the complexification $V^{\mathbb{C}}$.

We need some results about the decomposition of 3-forms on complex vector spaces with additional complex structure first.

Let $J$ be an automorphism of a six-dimensional real vector space $V$ satisfying $J^{2}=-I$. Furthermore, let $V^{\mathbb{C}}=V \oplus i V$ be the complexification of $V$. There is the standard decomposition $V^{\mathbb{C}}=V^{1,0} \oplus V^{0,1}$. Consider a nonzero form $\gamma$ of type $(3,0)$ on $V^{\mathbb{C}}$ and set

$$
\gamma_{0}=\operatorname{Re} \gamma, \quad \gamma_{1}=\operatorname{Im} \gamma
$$

For any $v_{1} \in V, v_{1}+i J v_{1} \in V^{0,1}$, and consequently $\gamma\left(i\left(v_{1}+i J v_{1}\right), v_{2}, v_{3}\right)=0$ for any $v_{2}, v_{3} \in V$. This implies that

$$
\gamma_{0}\left(i\left(v_{1}+i J v_{1}\right), v_{2}, v_{3}\right)=0 \quad \text { and } \quad \gamma_{1}\left(i\left(v_{1}+i J v_{1}\right), v_{2}, v_{3}\right)=0 .
$$

Thus,

$$
0=\gamma_{0}\left(i\left(v_{1}+i J v_{1}\right), v_{2}, v_{3}\right)=\gamma_{0}\left(i v_{1}, v_{2}, v_{3}\right)-\gamma_{0}\left(J v_{1}, v_{2}, v_{3}\right) .
$$

A similar argument with $\gamma_{1}$ leads to

$$
\gamma_{0}\left(i v_{1}, v_{2}, v_{3}\right)=\gamma_{0}\left(J v_{1}, v_{2}, v_{3}\right), \quad \gamma_{1}\left(i v_{1}, v_{2}, v_{3}\right)=\gamma_{1}\left(J v_{1}, v_{2}, v_{3}\right),
$$

for any $v_{1}, v_{2}, v_{3} \in V$. Moreover,

$$
\begin{aligned}
\gamma_{0}\left(w_{1}, w_{2}, w_{3}\right) & =\operatorname{Re}\left(-\gamma\left(i^{2} w_{1}, w_{2}, w_{3}\right)\right)=\operatorname{Re}\left(-i \gamma\left(i w_{1}, w_{2}, w_{3}\right)\right) \\
& =\operatorname{Im}\left(\gamma\left(i w_{1}, w_{2}, w_{3}\right)\right)=\gamma_{1}\left(i w_{1}, w_{2}, w_{3}\right),
\end{aligned}
$$

for any $w_{1}, w_{2}, w_{3} \in V^{\mathbb{C}}$, that is, $\gamma_{1}\left(w_{1}, w_{2}, w_{3}\right)=-\gamma_{0}\left(i w_{1}, w_{2}, w_{3}\right)$. Finally, 


$$
\begin{aligned}
\gamma_{0}\left(J v_{1}, v_{2}, v_{3}\right) & =\gamma_{0}\left(i v_{1}, v_{2}, v_{3}\right)=\operatorname{Re}\left(\gamma\left(i v_{1}, v_{2}, v_{3}\right)\right)=\operatorname{Re}\left(i \gamma\left(v_{1}, v_{2}, v_{3}\right)\right) \\
& =\operatorname{Re}\left(\gamma\left(v_{1}, i v_{2}, v_{3}\right)\right)=\operatorname{Re}\left(\gamma\left(v_{1}, J v_{2}, v_{3}\right)\right)=\gamma_{0}\left(v_{1}, J v_{2}, v_{3}\right)
\end{aligned}
$$

In a similar manner,

$$
\begin{aligned}
& \gamma_{0}\left(J v_{1}, v_{2}, v_{3}\right)=\gamma_{0}\left(v_{1}, J v_{2}, v_{3}\right)=\gamma_{0}\left(v_{1}, v_{2}, J v_{3}\right) \\
& \gamma_{1}\left(J v_{1}, v_{2}, v_{3}\right)=\gamma_{1}\left(v_{1}, J v_{2}, v_{3}\right)=\gamma_{1}\left(v_{1}, v_{2}, J v_{3}\right)
\end{aligned}
$$

that is, both forms $\gamma_{0}$ and $\gamma_{1}$ are pure with respect to the complex structure $J$.

We recall that a 3 -form $\omega$ on a vector space $V$ is called pure with respect to an automorphism $A$ of $V$ if and only if

$$
\omega\left(A X_{1}, X_{2}, X_{3}\right)=\omega\left(X_{1}, A X_{2}, X_{3}\right)=\omega\left(X_{1}, X_{2}, A X_{3}\right) \text { for all } X_{1}, X_{2}, X_{3} \in V \text {. }
$$

LEMMA 1. The real 3-forms $\gamma_{0} \mid V$ and $\gamma_{1} \mid V($ on $V)$ are multisymplectic.

Proof. Assume that $v_{1} \in V$ is a vector such that for any vectors $v_{2}, v_{3} \in V$ $\left(\gamma_{0} \mid V\right)\left(v_{1}, v_{2}, v_{3}\right)=0$ or equivalently $\gamma_{0}\left(v_{1}, v_{2}, v_{3}\right)=0$. There are uniquely determined vectors $w_{1}, w_{2}, w_{3} \in V^{1,0}$ such that

$$
v_{1}=w_{1}+\bar{w}_{1}, \quad v_{2}=w_{2}+\bar{w}_{2}, \quad v_{3}=w_{3}+\bar{w}_{3} .
$$

Then

$$
\begin{aligned}
0=\gamma_{0}\left(v_{1}, v_{2}, v_{3}\right) & =\operatorname{Re}\left(\gamma\left(w_{1}+\bar{w}_{1}, w_{2}+\bar{w}_{2}, w_{3}+\bar{w}_{3}\right)\right) \\
& =\operatorname{Re}\left(\gamma\left(w_{1}, w_{2}, w_{3}\right)\right)=\gamma_{0}\left(w_{1}, w_{2}, w_{3}\right)
\end{aligned}
$$

for a fixed $w_{1}$ and arbitrary $w_{2}, w_{3} \in V^{1,0}$. Because $i w_{2} \in V^{1,0}$,

$$
\gamma_{0}\left(i w_{1}, w_{2}, w_{3}\right)=\gamma_{0}\left(w_{1}, i w_{2}, w_{3}\right)=0 .
$$

Moreover, $\gamma_{1}\left(w, w^{\prime}, w^{\prime \prime}\right)=-\gamma_{0}\left(i w, w^{\prime}, w^{\prime \prime}\right)$ for any $w, w^{\prime}, w^{\prime \prime} \in V^{\mathbb{C}}$, giving

$$
\gamma_{1}\left(w_{1}, w_{2}, w_{3}\right)=-\gamma_{0}\left(i w_{1}, w_{2}, w_{3}\right)=0,
$$

for arbitrary $w_{2}, w_{3} \in V^{1,0}$. Thus,

$$
\gamma\left(w_{1}, w_{2}, w_{3}\right)=\gamma_{0}\left(w_{1}, w_{2}, w_{3}\right)+i \gamma_{1}\left(w_{1}, w_{2}, w_{3}\right)=0
$$

for arbitrary $w_{2}, w_{3} \in V^{1,0}$.

Because $\gamma$ is a nonzero complex 3-form on the complex three-dimensional vector space $V^{1,0}$, we find that $w_{1}=0$, and consequently $v_{1}=0$. This proves that the real 3-form $\gamma_{0} \mid V$ is multisymplectic. Similarly, the real 3-form $\gamma_{1} \mid V$ is also multisymplectic.

LEMMA 2. The forms $\gamma_{0} \mid V$ and $\gamma_{1} \mid V$ satisfy $\Delta\left(\gamma_{0} \mid V\right)=\{0\}$ and $\Delta\left(\gamma_{1} \mid V\right)=\{0\}$. 
PROOF. The complex 3-form $\gamma$ is decomposable, and therefore $\gamma \wedge \gamma=0$. This implies that, for any $w \in V^{\mathbb{C}},\left(\iota_{w} \gamma\right) \wedge\left(\iota_{w} \gamma\right)=0$. Similarly, for any $w \in V^{\mathbb{C}},\left(\iota_{w} \bar{\gamma}\right)$ $\wedge\left(\iota_{w} \bar{\gamma}\right)=0$. Obviously $\gamma_{0}=\frac{1}{2}(\gamma+\bar{\gamma})$. Let $v \in V$ be such that $\left(\iota_{v} \gamma_{0}\right) \wedge\left(\iota_{v} \gamma_{0}\right)=0$. Then

$$
0=\left(\iota_{v} \gamma_{0}\right) \wedge\left(\iota_{v} \gamma_{0}\right)=\frac{1}{4}\left(\iota_{v} \gamma+\iota_{v} \bar{\gamma}\right) \wedge\left(\iota_{v} \gamma+\iota_{v} \bar{\gamma}\right)=\frac{1}{2}\left(\iota_{v} \gamma\right) \wedge\left(\iota_{v} \bar{\gamma}\right) .
$$

But $\iota_{v} \gamma$ is a form of type $(2,0)$ and $\iota_{v} \bar{\gamma}$ a form of type $(0,2)$. Consequently the last wedge product vanishes if and only if either $\iota_{v} \gamma=0$ or $\iota_{v} \bar{\gamma}=0$. By virtue of the preceding lemma, this implies that $v=0$.

Lemma 2 shows that both forms $\gamma_{0} \mid V$ and $\gamma_{1} \mid V$ are of complex type. As a final result of this type we get the following.

COROllary 3. Let $\gamma$ be a 3-form on $V^{\mathbb{C}}$ of type $(3,0)$. Then the real 3-forms $(\operatorname{Re} \gamma) \mid V$ and $(\operatorname{Im} \gamma) \mid V$ on $V$ are multisymplectic and of complex type.

Let $\omega$ be a 3-form on $V$ such that $\Delta(\omega)=\{0\}$. This means that for any $v \in V, v \neq 0$, $\left(\iota_{v} \omega\right) \wedge\left(\iota_{v} \omega\right) \neq 0$. This implies that $\operatorname{rank} \iota_{v} \omega \geq 4$. On the other hand, obviously rank $\iota_{v} \omega \leq 4$. Consequently, for any $v \neq 0, \operatorname{rank} \iota_{v} \omega=4$. Thus the kernel $K\left(\iota_{v} \omega\right)$ of the 2-form $\iota_{v} \omega$ has dimension two. Moreover, $v \in K\left(\iota_{v} \omega\right)$. Now we fix a nonzero 6-form on $\theta$ on $V$. For any $v \in V$, there exists a unique vector $Q(v) \in V$ such that

$$
\left(\iota_{v} \omega\right) \wedge \omega=\iota_{Q(v)} \theta .
$$

The mapping $Q: V \rightarrow V$ is obviously a homomorphism. If $v \neq 0$ then $\left(\iota_{v} \omega\right) \wedge \omega \neq 0$, and $Q$ is an automorphism. It is also obvious that if $v \neq 0$, then the vectors $v$ and $Q(v)$ are linearly independent (by applying $\iota_{v}$ to the last equality). We evaluate $\iota_{Q(v)}$ on the last equality and obtain

$$
\begin{gathered}
\left(\iota_{Q(v)} \iota_{v} \omega\right) \wedge \omega+\left(\iota_{v} \omega\right) \wedge\left(\iota_{Q(v)} \omega\right)=0 \\
-\left(\iota_{v} \iota_{Q(v)} \omega\right) \wedge \omega+\left(\iota_{v} \omega\right) \wedge\left(\iota_{Q(v)} \omega\right)=0 \\
-\iota_{v}\left[\left(\iota_{Q(v)} \omega\right) \wedge \omega\right]+2\left(\iota_{v} \omega\right) \wedge\left(\iota_{Q(v)} \omega\right)=0 .
\end{gathered}
$$

Now apply $\iota_{v}$ to the last equality:

$$
\left(\iota_{v} \omega\right) \wedge\left(\iota_{v} \iota_{Q(v)} \omega\right)=0 .
$$

If the 1-form $\iota_{v} \iota_{Q(v)} \omega$ was not zero then there would exist a 1-form $\sigma$ such that $\iota_{v} \omega=\sigma \wedge \iota_{v} \iota Q(v) \omega$, which would give

$$
\left(\iota_{v} \omega\right) \wedge\left(\iota_{v} \omega\right)=\sigma \wedge \iota_{v} \iota_{Q(v)} \omega \wedge \sigma \wedge \iota_{v} \iota_{Q(v)} \omega=0,
$$

which is a contradiction. Thus, we have proved the following lemma.

Lemma 4. For any $v \in V, \iota_{Q(v)} \iota_{v} \omega=0$, that is, $Q(v) \in K\left(\iota_{v} \omega\right)$. 
This lemma shows that if $v \neq 0$, then $K\left(\iota_{v} \omega\right)=[v, Q(v)]$. Applying $\iota_{Q(v)}$ to the equality $\left(\iota_{v} \omega\right) \wedge \omega=\iota_{Q(v)} \theta$ and using the last lemma, we easily obtain the following result.

LEMMA 5. For any $v \in V,\left(\iota_{v} \omega\right) \wedge\left(\iota_{Q(v)} \omega\right)=0$.

Lemma 4 shows that $v \in K\left(\iota_{Q(v)} \omega\right)$. Because $v$ and $Q(v)$ are linearly independent, we can see that

$$
K\left(\iota_{Q}(v) \omega\right)=[v, Q(v)]=K\left(\iota_{v} \omega\right) .
$$

If $v \neq 0$, then $Q^{2}(v) \in K\left(\iota_{Q}(v) \omega\right)$, and consequently there exist $a(v), b(v) \in \mathbb{R}$ such that

$$
Q^{2}(v)=a(v) v+b(v) Q(v) .
$$

For any $v \in V$,

$$
\left(\iota_{Q(v)} \omega\right) \wedge \omega=\iota_{Q^{2}(v)} \theta
$$

Assume that $v \neq 0$. Then

$$
\left(\iota_{Q(v)} \omega\right) \wedge \omega=a(v) \iota_{v} \theta+b(v) \iota_{Q(v)} \theta,
$$

and, applying $\iota_{v}$, we obtain $b(v) \iota_{v} \iota_{Q(v)} \theta=0$, which shows that $b(v)=0$ for any $v \neq 0$. Consequently, $Q^{2}(v)=a(v) v$ for any $v \neq 0$.

LEMMA 6. Let $A: V \rightarrow V$ be an automorphism, and $a: V \backslash\{0\} \rightarrow \mathbb{R}$ a function such that

$$
A(v)=a(v) v, \quad \text { for any } v \neq 0 .
$$

Then the function a is constant.

Proof. The condition on $A$ means that every vector $v$ of $V$ is an eigenvector of $A$ with the eigenvalue $a(v)$. But the eigenvalues of two different vectors have to be the same otherwise their sum would not be an eigenvector.

Applying Lemma 6 on $Q^{2}$ gives $Q^{2}=a I$. If $a>0$, then $V=V^{+} \oplus V^{-}$, and

$$
Q v=\sqrt{a} v \quad \text { for } v \in V^{+}, \quad Q v=-\sqrt{a} v \text { for } v \in V^{-} .
$$

At least one of the subspaces $V^{+}$and $V^{-}$is nontrivial. Assume, for example, that $V^{+} \neq\{0\}$. Then there exist $v \in V^{+}, v \neq 0$, and $Q v=\sqrt{a} v$, which is a contradiction because the vectors $v$ and $Q v$ are linearly independent. This proves that $a<0$. We can now see that the automorphisms

$$
J_{+}=\frac{1}{\sqrt{-a}} Q \quad \text { and } \quad J_{-}=-\frac{1}{\sqrt{-a}} Q
$$

satisfy $J_{+}^{2}=-I$ and $J_{-}^{2}=-I$, that is, they define complex structures on $V$ and $J_{-}=-J_{+}$. Setting

$$
\theta_{+}=\sqrt{-a} \theta, \quad \theta_{-}=-\sqrt{-a} \theta
$$


we obtain

$$
\left(\iota_{v} \omega\right) \wedge \omega=\iota_{J_{+} v} \theta_{+}, \quad\left(\iota_{v} \omega\right) \wedge \omega=\iota_{J_{-} v} \theta_{-} .
$$

In what follows we use the notation $J=J_{+}$. Results which are valid for $J_{+}$also hold for $J_{-}$.

PROPOSITION 7. There exists a unique (up to sign) complex structure $J$ on $V$ such that the form $\omega$ satisfies the relation

$$
\omega\left(J v_{1}, v_{2}, v_{3}\right)=\omega\left(v_{1}, J v_{2}, v_{3}\right)=\omega\left(v_{1}, v_{2}, J v_{3}\right) \quad \text { for any } v_{1}, v_{2}, v_{3} \in V .
$$

PROOF. We shall prove first that the complex structure $J$ defined above satisfies the relation. By virtue of Lemma 4 for any $v, v^{\prime} \in V, \omega\left(v, J v, v^{\prime}\right)=0$. Therefore,

$$
\begin{aligned}
0=\omega\left(v_{1}+v_{2}, J\left(v_{1}+v_{2}\right), v_{3}\right) & =\omega\left(v_{1}, J v_{2}, v_{3}\right)+\omega\left(v_{2}, J v_{1}, v_{3}\right) \\
& =-\omega\left(J v_{1}, v_{2}, v_{3}\right)+\omega\left(v_{1}, J v_{2}, v_{3}\right),
\end{aligned}
$$

which gives

$$
\omega\left(J v_{1}, v_{2}, v_{3}\right)=\omega\left(v_{1}, J v_{2}, v_{3}\right) .
$$

Obviously, the opposite complex structure $-J$ satisfies the same relation. We prove that there is no other complex structure with the same property. Let $\tilde{J}$ be a complex structure on $V$ satisfying the above relation. Set $A=\tilde{J} J^{-1}$. Then

$$
\begin{aligned}
\omega\left(v_{1}, A v_{2}, A v_{3}\right)=\omega\left(v_{1}, \tilde{J} J v_{2}, \tilde{J} J v_{3}\right) & =\omega\left(v_{1}, J v_{2}, \tilde{J}^{2} J v_{3}\right)=-\omega\left(v_{1}, J v_{2}, J v_{3}\right) \\
& =-\omega\left(v_{1}, v_{2}, J^{2} v_{3}\right)=\omega\left(v_{1}, v_{2}, v_{3}\right) .
\end{aligned}
$$

Any automorphism $A$ satisfying this identity is $\pm I$. In fact the identity means that $A$ is an automorphism of the 2 -form $\iota_{v} \omega$. Consequently, $A$ preserves the kernel $K\left(\iota_{v} \omega\right)=[v, J v]$. On the other hand, it is obvious that any subspace of the form $[v, J v]$ is the kernel of $\iota_{v} \omega$. Considering $V$ as a complex vector space with the complex structure $J$, we can say that every one-dimensional complex subspace is the kernel of the 2-form $\iota_{v} \omega$ for some $v \in V, v \neq 0$, and consequently is invariant under the automorphism $A$. As in Lemma 6 , we conclude that $A=\lambda I, \lambda \in \mathbb{C}$. If we write $\lambda=\lambda_{0}+i \lambda_{1}$, then $A=\lambda_{0} I+\lambda_{1} J$ and

$$
\begin{aligned}
& \omega\left(v_{1}, v_{2}, v_{3}\right)= \omega\left(v_{1}, A v_{2}, A v_{3}\right) \\
&= \omega\left(v_{1}, \lambda_{0} v_{2}+\lambda_{1} J v_{2}, \lambda_{0} v_{3}+\lambda_{1} J v_{3}\right) \\
&= \lambda_{0}^{2} \omega\left(v_{1}, v_{2}, v_{3}\right)+\lambda_{0} \lambda_{1} \omega\left(v_{1}, v_{2}, J v_{3}\right)+\lambda_{0} \lambda_{1} \omega\left(v_{1}, J v_{2}, v_{3}\right) \\
&+\lambda_{1}^{2} \omega\left(v_{1}, J v_{2}, J v_{3}\right), \\
&\left(\lambda_{0}^{2}-\lambda_{1}^{2}-1\right) \omega\left(v_{1}, v_{2}, v_{3}\right)+2 \lambda_{0} \lambda_{1} \omega\left(v_{1}, v_{2}, J v_{3}\right)=0 .
\end{aligned}
$$

We use this last equation together with one obtained by writing $J v_{3}$ instead of $v_{3}$. In this way we get the system

$$
\begin{gathered}
\left(\lambda_{0}^{2}-\lambda_{1}^{2}-1\right) \omega\left(v_{1}, v_{2}, v_{3}\right)+2 \lambda_{0} \lambda_{1} \omega\left(v_{1}, v_{2}, J v_{3}\right)=0 \\
-2 \lambda_{0} \lambda_{1} \omega\left(v_{1}, v_{2}, v_{3}\right)+\left(\lambda_{0}^{2}-\lambda_{1}^{2}-1\right) \omega\left(v_{1}, v_{2}, J v_{3}\right)=0
\end{gathered}
$$


Because it has a nontrivial solution,

$$
\left|\begin{array}{cc}
\lambda_{0}^{2}-\lambda_{1}^{2}-1 & 2 \lambda_{0} \lambda_{1} \\
-2 \lambda_{0} \lambda_{1} & \lambda_{0}^{2}-\lambda_{1}^{2}-1
\end{array}\right|=0 .
$$

It is easy to verify that the solution of the last equation is $\lambda_{0}= \pm 1$ and $\lambda_{1}=0$. This finishes the proof.

We shall now consider the vector space $V$, together with a complex structure $J$ and a 3-form $\omega$ on $V$ which is pure with respect to this complex structure. First, define a real 3-form $\gamma_{0}$ on $V^{\mathbb{C}}$. Set

$$
\begin{gathered}
\gamma_{0}\left(v_{1}, v_{2}, v_{3}\right)=\omega\left(v_{1}, v_{2}, v_{3}\right) \\
\gamma_{0}\left(i v_{1}, v_{2}, v_{3}\right)=\omega\left(J v_{1}, v_{2}, v_{3}\right) \\
\gamma_{0}\left(i v_{1}, i v_{2}, v_{3}\right)=\omega\left(J v_{1}, J v_{2}, v_{3}\right) \\
\gamma_{0}\left(i v_{1}, i v_{2}, i v_{3}\right)=\omega\left(J v_{1}, J v_{2}, J v_{3}\right)
\end{gathered}
$$

for $v_{1}, v_{2}, v_{3} \in V$. Then $\gamma_{0}$ extends uniquely to a real 3-form on $V^{\mathbb{C}}$. We can easily verify that

$$
\gamma_{0}\left(i w_{1}, w_{2}, w_{3}\right)=\gamma_{0}\left(w_{1}, i w_{2}, w_{3}\right)=\gamma_{0}\left(w_{1}, w_{2}, i w_{3}\right),
$$

for any $w_{1}, w_{2}, w_{3} \in V^{\mathbb{C}}$. Furthermore, set

$$
\gamma_{1}\left(w_{1}, w_{2}, w_{3}\right)=-\gamma_{0}\left(i w_{1}, w_{2}, w_{3}\right) \text { for } w_{1}, w_{2}, w_{3} \in V^{\mathbb{C}} .
$$

It is obvious that $\gamma_{1}$ is a real 3-form satisfying

$$
\gamma_{1}\left(i w_{1}, w_{2}, w_{3}\right)=\gamma_{1}\left(w_{1}, i w_{2}, w_{3}\right)=\gamma_{1}\left(w_{1}, w_{2}, i w_{3}\right),
$$

for any $w_{1}, w_{2}, w_{3} \in V^{\mathbb{C}}$. Now define

$$
\gamma\left(w_{1}, w_{2}, w_{3}\right)=\gamma_{0}\left(w_{1}, w_{2}, w_{3}\right)+i \gamma_{1}\left(w_{1}, w_{2}, w_{3}\right) \text { for } w_{1}, w_{2}, w_{3} \in V^{\mathbb{C}} .
$$

It is obvious that $\gamma$ is skew-symmetric and 3-linear over $\mathbb{R}$ and has complex values. Moreover,

$$
\begin{aligned}
\gamma\left(i w_{1}, w_{2}, w_{3}\right) & =\gamma_{0}\left(i w_{1}, w_{2}, w_{3}\right)+i \gamma_{1}\left(i w_{1}, w_{2}, w_{3}\right) \\
& =-\gamma_{1}\left(w_{1}, w_{2}, w_{3}\right)-i \gamma_{0}\left(i^{2} w_{1}, w_{2}, w_{3}\right) \\
& =-\gamma_{1}\left(w_{1}, w_{2}, w_{3}\right)+i \gamma_{0}\left(w_{1}, w_{2}, w_{3}\right) \\
& =i\left[\gamma_{0}\left(w_{1}, w_{2}, w_{3}\right)+i \gamma_{1}\left(w_{1}, w_{2}, w_{3}\right)\right]=i \gamma\left(w_{1}, w_{2}, w_{3}\right),
\end{aligned}
$$

which proves that $\gamma$ is a complex 3 -form on $V^{\mathbb{C}}$. We now prove that $\gamma$ is a form of type $(3,0)$. Obviously, it suffices to prove that for $v_{1}+i J v_{1} \in V^{0,1}$ and $v_{2}, v_{3} \in V$, $\gamma\left(v_{1}+i J v_{1}, v_{2}, v_{3}\right)=0$. Indeed,

$$
\begin{aligned}
\gamma\left(v_{1}\right. & \left.+i J v_{1}, v_{2}, v_{3}\right)=\gamma\left(v_{1}, v_{2}, v_{3}\right)+i \gamma\left(J v_{1}, v_{2}, v_{3}\right) \\
& =\gamma_{0}\left(v_{1}, v_{2}, v_{3}\right)+i \gamma_{1}\left(v_{1}, v_{2}, v_{3}\right)+i \gamma_{0}\left(J v_{1}, v_{2}, v_{3}\right)-\gamma_{1}\left(J v_{1}, v_{2}, v_{3}\right) \\
& =\gamma_{0}\left(v_{1}, v_{2}, v_{3}\right)-i \gamma_{0}\left(i v_{1}, v_{2}, v_{3}\right)+i \gamma_{0}\left(J v_{1}, v_{2}, v_{3}\right)+\gamma_{0}\left(i J v_{1}, v_{2}, v_{3}\right) .
\end{aligned}
$$


Now $\gamma_{0}\left(i J v_{1}, v_{2}, v_{3}\right)=\omega\left(J^{2} v_{1}, v_{2}, v_{3}\right)=-\omega\left(v_{1}, v_{2}, v_{3}\right)=-\gamma_{0}\left(v_{1}, v_{2}, v_{3}\right)$ and the real part of the last expression is zero. Furthermore, $\gamma_{0}\left(J v_{1}, v_{2}, v_{3}\right)$ $=\omega\left(J v_{1}, v_{2}, v_{3}\right)=\gamma_{0}\left(i v_{1}, v_{2}, v_{3}\right)$ and the complex part of the expression is also zero. Now we easily obtain the following proposition.

PROposition 8. Let $\omega$ be a real 3-form on $V$ satisfying $\Delta(\omega)=\{0\}$, and let $J$ be a complex structure on $V$ (one of the two) such that

$$
\omega\left(J v_{1}, v_{2}, v_{3}\right)=\omega\left(v_{1}, J v_{2}, v_{3}\right)=\omega\left(v_{1}, v_{2}, J v_{3}\right)
$$

Then there exists on $V^{\mathbb{C}}$ a unique complex 3-form $\gamma$ of type $(3,0)$ such that:

$$
\omega=(\operatorname{Re} \gamma) \mid V \text {. }
$$

REMARK. The complex structure $J$ on $V$ can also be introduced by means of Hitchin's invariant $\lambda$, as in $[\mathrm{H}]$. Forms of complex type form an open subset $U$ in $\Lambda^{3} V^{*}$. Hitchin has shown that this manifold is also endowed with an almost complex structure which is integrable. Hitchin uses the following method to introduce an almost complex structure on $U$. One regards $U \subset \Lambda^{3} V^{*}$ as a symplectic manifold (let $\theta$ be a fixed element in $\Lambda^{6} V^{*}$; one defines the symplectic form $\Theta$ on $\Lambda^{3} V^{*}$ by the equation $\left.\omega_{1} \wedge \omega_{2}=\Theta\left(\omega_{1}, \omega_{2}\right) \theta\right)$. Then the derivative of the Hamiltonian vector field corresponding to the function $\sqrt{-\lambda(\omega)}$ on $U$ gives an integrable almost complex structure on $U$.

There is another way of introducing this almost complex structure on $U$. Given a 3form $\omega \in U$, choose the complex structure $J_{\omega}$ on $V$ (one of the two), whose existence is guaranteed by the Proposition 7. Then we define endomorphisms $A_{J_{\omega}}$ and $D_{J_{\omega}}$ of $\Lambda^{k} V^{*}$ by

$$
\begin{gathered}
\left(A_{J_{\omega}} \Omega\right)\left(v_{1}, \ldots, v_{k}\right)=\Omega\left(J_{\omega} v_{1}, \ldots, J_{\omega} v_{k}\right) \\
\left(D_{J_{\omega}} \Omega\right)\left(v_{1}, \ldots, v_{k}\right)=\sum_{i=1}^{k} \Omega\left(v_{1}, \ldots, v_{i-1}, J_{\omega} v_{i}, v_{i+1}, \ldots, v_{k}\right) .
\end{gathered}
$$

Then $A_{J_{\omega}}$ is an automorphism of $\Lambda V^{*}$ and $D_{J_{\omega}}$ is a derivation of $\Lambda V^{*}$. If $k=3$ then the automorphism $-\frac{1}{2}\left(A_{J_{\omega}}+D_{J_{\omega}}\right)$ of $\Lambda^{3} V^{*}\left(=T_{\omega} U\right)$ gives a complex structure on $U$ and coincides with that of Hitchin.

\section{Three-forms of complex type on manifolds}

We use facts from the previous section to obtain some global results on 3-forms on six-dimensional manifolds. We denote the real vector fields on a (real) manifold $M$ by $X, Y, Z$ and the complex vector fields on $M$ by $V, W$. Here $\mathfrak{X}(M)$ stands for the set of all (real) vector fields on $M$ and $\mathfrak{X}^{\mathbb{C}}(M)$ means all of the complex vector fields on $M$.

A 3-form $\omega$ on $M$ is called a form of complex type if, for every $x \in M, \Delta\left(\omega_{x}\right)=\{0\}$. Let $\omega$ be a form of complex type on $M$ and let $U \subset M$ be an open orientable 
submanifold. Then there exists an everywhere nonzero differentiable 6-form on $U$. In each $T_{x} M, x \in U$, construct $J_{-}$and $J_{+}$as in Proposition 7. The construction is evidently smooth on $U$. Thus, we obtain the following lemma.

LEMMA 9. Let $\omega$ be a form of complex type on $M$ and let $U \subset M$ be an orientable open submanifold. Then there exist two differentiable almost complex structures $J_{+}$ and $J_{-}$on $U$ such that, for any vector fields $X_{1}, X_{2}, X_{3}$ :

(i) $J_{+}+J_{-}=0$

(ii) $\omega\left(J_{+} X_{1}, X_{2}, X_{3}\right)=\omega\left(X_{1}, J_{+} X_{2}, X_{3}\right)=\omega\left(X_{1}, X_{2}, J_{+} X_{3}\right)$;

(iii) $\omega\left(J_{-} X_{1}, X_{2}, X_{3}\right)=\omega\left(X_{1}, J_{-} X_{2}, X_{3}\right)=\omega\left(X_{1}, X_{2}, J_{-} X_{3}\right)$.

At each point $x \in M$ consider a one-dimensional subspace of the space $\mathcal{T}_{1 x}^{1}(M)$ of tensors of type $(1,1)$ at $x$ generated by the tensors $J_{+x}$ and $J_{-x}$. The above considerations show that it is a one-dimensional subbundle $\mathcal{J} \subset \mathcal{T}_{1}^{1}(M)$.

LEMMA 10. The one-dimensional vector bundles $\mathcal{J}$ and $\Lambda^{6} T^{*}(M)$ are isomorphic.

Proof. Choose a Riemannian metric $g_{0}$ on $T M$. If $x \in M$ and $v, v^{\prime} \in T_{x} M$, define a Riemannian metric $g$ by the formula

$$
g\left(v, v^{\prime}\right)=g_{0}\left(v, v^{\prime}\right)+g_{0}\left(J_{+} v, J_{+} v^{\prime}\right)=g_{0}\left(v, v^{\prime}\right)+g_{0}\left(J_{-} v, J_{-} v^{\prime}\right) .
$$

It is obvious that, for any $v, v^{\prime} \in T_{x} M$,

$$
g\left(J_{+} v, J_{+} v^{\prime}\right)=g\left(v, v^{\prime}\right), \quad g\left(J_{-} v, J_{-} v^{\prime}\right)=g\left(v, v^{\prime}\right) .
$$

Now define

$$
\sigma_{+}\left(v, v^{\prime}\right)=g\left(J_{+} v, v^{\prime}\right), \quad \sigma_{-}\left(v, v^{\prime}\right)=g\left(J_{-} v, v^{\prime}\right)
$$

It is easy to verify that $\sigma_{+}$and $\sigma_{-}$are nonzero 2 -forms on $T_{x} M$ satisfying $\sigma_{+}+\sigma_{-}=0$.

Define an isomorphism $h: \mathcal{J} \rightarrow \Lambda^{6} T^{*} M$. Let $x \in M$ and $A \in \mathcal{J}_{x}$. Write

$$
A=a J_{+}, \quad A=-a J_{-}
$$

and set

$$
h A=a \sigma_{+} \wedge \sigma_{+} \wedge \sigma_{+}=-a \sigma_{-} \wedge \sigma_{-} \wedge \sigma_{-} .
$$

COROllary 11. There exist two almost complex structures $J_{+}$and $J_{-}$on $M$ such that: 
(i) $J_{+}+J_{-}=0$;

(ii) $\omega\left(J_{+} X_{1}, X_{2}, X_{3}\right)=\omega\left(X_{1}, J_{+} X_{2}, X_{3}\right)=\omega\left(X_{1}, X_{2}, J_{+} X_{3}\right)$;

(iii) $\omega\left(J_{-} X_{1}, X_{2}, X_{3}\right)=\omega\left(X_{1}, J_{-} X_{2}, X_{3}\right)=\omega\left(X_{1}, X_{2}, J_{-} X_{3}\right)$;

for any vector fields $X_{1}, X_{2}, X_{3}$, if and only if the manifold $M$ is orientable.

Hence the assertions in the rest of the paper can be simplified correspondingly if $M$ is an orientable manifold.

LEMMA 12. Let $J$ be an almost complex structure on $M$ such that for any vector fields $X_{1}, X_{2}, X_{3} \in \mathfrak{X}(M)$,

$$
\omega\left(J X_{1}, X_{2}, X_{3}\right)=\omega\left(X_{1}, J X_{2}, X_{3}\right)=\omega\left(X_{1}, X_{2}, J X_{3}\right) .
$$

If $\nabla$ is a linear connection on $M$ such that $\nabla \omega=0$, then also $\nabla J=0$.

PROOF. Let $Y \in \mathfrak{X}(M)$, and consider the covariant derivative $\nabla_{Y}$. Then

$$
\begin{aligned}
0= & \left(\nabla_{Y} \omega\right)\left(J X_{1}, X_{2}, X_{3}\right)=Y\left(\omega\left(J X_{1}, X_{2}, X_{3}\right)-\omega\left(\left(\nabla_{Y} J\right) X_{1}, X_{2}, X_{3}\right)\right. \\
& -\omega\left(J \nabla_{Y} X_{1}, X_{2}, X_{2}\right)-\omega\left(J X_{1}, \nabla_{Y} X_{2}, X_{3}\right)-\omega\left(J X_{1}, X_{2}, \nabla_{Y} X_{3}\right) \\
0= & \left(\nabla_{Y} \omega\right)\left(X_{1}, J X_{2}, X_{3}\right)=Y\left(\omega\left(J X_{1}, X_{2}, X_{3}\right)-\omega\left(\nabla_{Y} X_{1}, J X_{2}, X_{3}\right)\right. \\
& -\omega\left(X_{1},\left(\nabla_{Y} J\right) X_{2}, X_{3}\right)-\omega\left(X_{1}, J \nabla_{Y} X_{2}, X_{3}\right)-\omega\left(X_{1}, J X_{2}, \nabla_{Y} X_{3}\right) .
\end{aligned}
$$

Because the above expressions are equal, it easily follows that

$$
\omega\left(\left(\nabla_{Y} J\right) X_{1}, X_{2}, X_{3}\right)=\omega\left(X_{1},\left(\nabla_{Y} J\right) X_{2}, X_{3}\right) .
$$

Write $A=\nabla_{Y} J$. Extending the above equality in the obvious way gives

$$
\omega\left(A X_{1}, X_{2}, X_{3}\right)=\omega\left(X_{1}, A X_{2}, X_{3}\right)=\omega\left(X_{1}, X_{2}, A X_{3}\right) .
$$

Moreover $J^{2}=-I$, and applying $\nabla_{Y}$ to this equality gives

$$
A J+J A=0 .
$$

We know that $K\left(\iota_{X} \omega\right)=[X, J X]$. Furthermore,

$$
\omega\left(X, A X, X^{\prime}\right)=\omega\left(X, X, A X^{\prime}\right)=0, \quad \omega\left(X, A J X, X^{\prime}\right)=\omega\left(X, J X, A X^{\prime}\right)=0,
$$

which shows that $A$ preserves the distribution $[X, J X]$. By the very same arguments as in Proposition 7 we can see that $A=\lambda_{0} I+\lambda_{1} J$. Consequently

$$
\begin{gathered}
\left(\lambda_{0} I+\lambda_{1} J\right) J+J\left(\lambda_{0} I+\lambda_{1} J\right)=0 \\
-2 \lambda_{1} I+2 \lambda_{0} J=0
\end{gathered}
$$

which implies that $\lambda_{0}=\lambda_{1}=0$. Thus, $\nabla_{Y} J=A=0$. 
The statement of the previous lemma can to some extent be reversed, giving the following theorem.

THEOREM 13. Let $\omega$ be a real 3-form on a six-dimensional differentiable manifold $M$ satisfying $\Delta\left(\omega_{x}\right)=\{0\}$ for any $x \in M$. Let $J$ be an almost complex structure on $M$ such that for any vector fields $X_{1}, X_{2}, X_{3} \in \mathfrak{X}(M)$,

$$
\omega\left(J X_{1}, X_{2}, X_{3}\right)=\omega\left(X_{1}, J X_{2}, X_{3}\right)=\omega\left(X_{1}, X_{2}, J X_{3}\right) .
$$

Then there exists a symmetric connection $\tilde{\nabla}$ on $M$ such that $\tilde{\nabla} \omega=0$ if and only if the following conditions are satisfied:

(i) $d \omega=0$;

(ii) the almost complex structure $J$ is integrable.

PROOF. First, we prove that the integrability of the structure $J$ and the fact that $\omega$ is closed imply the existence of a symmetric connection with respect to which $\omega$ is parallel.

For any connection $\nabla$ on $M$ we denote its complexification using the same symbol. That is, we set

$$
\nabla_{X_{0}+i X_{1}}\left(Y_{0}+i Y_{1}\right)=\left(\nabla_{X_{0}} Y_{0}-\nabla_{X_{1}} Y_{1}\right)+i\left(\nabla_{X_{0}} Y_{1}+\nabla_{X_{1}} Y_{0}\right)
$$

Assume that there exists a symmetric connection $\stackrel{\circ}{\nabla}$ such that $\stackrel{\circ}{\nabla} J=0$. Consider a 3 -form $\gamma$ of type $(3,0)$ such that $(\operatorname{Re} \gamma) \mid T M=\omega$. Our next aim is to try to find a symmetric connection

$$
\nabla_{V} W=\stackrel{\circ}{\nabla}_{V} W+Q(V, W)
$$

satisfying $\nabla_{V} \gamma=0$. Obviously, the connection $\nabla$ is symmetric if and only if

$$
Q(V, W)=Q(W, V) .
$$

Moreover, $\nabla_{V} \gamma=0$ suggests that $\nabla J=0$. The equality

$$
\begin{aligned}
0=\left(\nabla_{V} J\right) W & =\nabla_{V}(J W)-J \nabla_{V} W \\
& =\stackrel{\circ}{\nabla}_{V}(J W)+Q(V, J W)-J \stackrel{\circ}{\nabla}_{V} W-J Q(V, W)
\end{aligned}
$$

shows that we should require

$$
Q(J V, W)=Q(V, J W)=J Q(V, W) .
$$

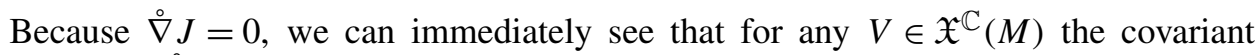
derivative $\stackrel{\circ}{\nabla}_{V} \gamma$ is again a form of type $(3,0)$. Consequently there exists a uniquely determined complex 1 -form $\rho$ such that

$$
\stackrel{\circ}{\nabla}_{V} \gamma=\rho(V) \gamma
$$


Then

$$
\begin{aligned}
\left(\nabla_{V} \gamma\right) & \left(W_{1}, W_{2}, W_{3}\right) \\
= & V\left(\gamma\left(W_{1}, W_{2}, W_{3}\right)\right)-\gamma\left(\nabla_{V} W_{1}, W_{2}, W_{3}\right) \\
& -\gamma\left(W_{1}, \nabla_{V} W_{2}, W_{3}\right)-\gamma\left(W_{1}, W_{2}, \nabla_{V} W_{3}\right) \\
= & V\left(\gamma\left(W_{1}, W_{2}, W_{3}\right)\right)-\gamma\left(\stackrel{\circ}{\nabla}_{V} W_{1}, W_{2}, W_{3}\right) \\
& -\gamma\left(W_{1}, \stackrel{\circ}{V}_{V} W_{2}, W_{3}\right)-\gamma\left(W_{1}, W_{2}, \stackrel{\circ}{\nabla}_{V} W_{3}\right) \\
& -\gamma\left(Q\left(V, W_{1}\right), W_{2}, W_{3}\right)-\gamma\left(W_{1}, Q\left(V, W_{2}\right), W_{3}\right) \\
& -\gamma\left(W_{1}, W_{2}, Q\left(V, W_{3}\right)\right) \\
= & \rho(V) \gamma\left(W_{1}, W_{2}, W_{3}\right) \\
& -\gamma\left(Q\left(V, W_{1}\right), W_{2}, W_{3}\right)-\gamma\left(W_{1}, Q\left(V, W_{2}\right), W_{3}\right) \\
& -\gamma\left(W_{1}, W_{2}, Q\left(V, W_{3}\right)\right) .
\end{aligned}
$$

In other words $\nabla_{V} \gamma=0$ if and only if

$$
\begin{aligned}
\rho(V) \gamma\left(W_{1}, W_{2}, W_{3}\right)= & \gamma\left(Q\left(V, W_{1}\right), W_{2}, W_{3}\right) \\
& +\gamma\left(W_{1}, Q\left(V, W_{2}\right), W_{3}\right)+\gamma\left(W_{1}, W_{2}, Q\left(V, W_{3}\right)\right) .
\end{aligned}
$$

SublemMA. If $d \gamma=0$, then $\rho$ is a form of type $(1,0)$.

Proof. Let $V_{1} \in T^{0,1}(M)$. Because $\stackrel{\circ}{\nabla}$ is symmetric $d \gamma=-\mathcal{A}(\stackrel{\circ}{\nabla} \gamma)$, where $\mathcal{A}$ denotes the alternation. We obtain

$$
\begin{aligned}
0 & =-4 !(d \gamma)\left(V_{1}, V_{2}, V_{3}, V_{4}\right) \\
& =\sum_{\pi} \operatorname{sign}(\pi)\left(\stackrel{\circ}{\nabla}_{V_{\pi 1}} \gamma\right)\left(V_{\pi 2}, V_{\pi 3}, V_{\pi 4}\right)+\sum_{\tau} \operatorname{sign}(\tau)\left(\stackrel{\circ}{\nabla}_{V_{1}} \gamma\right)\left(V_{\tau 2}, V_{\tau 3}, V_{\tau 4}\right) \\
& =3 !\left(\stackrel{\circ}{\nabla}_{V_{1}} \gamma\right)\left(V_{2}, V_{3}, V_{4}\right) \\
& =3 ! \rho\left(V_{1}\right) \gamma\left(V_{2}, V_{3}, V_{4}\right) .
\end{aligned}
$$

The first sum is taken over all permutations $\pi$ satisfying $\pi 1>1$, and the second is taken over all permutations of the set $\{2,3,4\}$. The first sum obviously vanishes, and $\rho\left(V_{1}\right)=0$. This finishes the proof.

We now set

$$
Q(V, W)=\frac{1}{8}[\rho(V) W-\rho(J V) J W+\rho(W) V-\rho(J W) J V] .
$$

It is easy to see that $Q(J V, W)=Q(V, J W)=J Q(V, W)$. For $V, W_{1}, W_{2}, W_{3}$ $\in T^{1,0}(M)$ we can compute

$$
\begin{aligned}
8 \gamma\left(Q\left(V, W_{1}\right), W_{2}, W_{3}\right)= & \gamma\left(\rho(V) W_{1}-\rho(J V) J W_{1}+\rho\left(W_{1}\right) V\right. \\
& \left.-\rho\left(J W_{1}\right) J V, W_{2}, W_{3}\right) \\
= & \gamma\left(2 \rho(V) W_{1}+2 \rho\left(W_{1}\right) V, W_{2}, W_{3}\right) \\
= & 2 \rho(V) \gamma\left(W_{1}, W_{2}, W_{3}\right)+2 \rho\left(W_{1}\right) \gamma\left(V, W_{2}, W_{3}\right),
\end{aligned}
$$


using for $V \in T^{(1,0)}(M)$ the fact that $\rho(J V)=i \rho(V)$ and $\gamma\left(J V, V^{\prime}, V^{\prime \prime}\right)$ $=i \gamma\left(V, V^{\prime}, V^{\prime \prime}\right)$, since $\gamma$ is of type $(3,0)$ and $\rho$ of type $(1,0)$.

Similarly we can compute $\gamma\left(W_{1}, Q\left(V, W_{2}\right), W_{3}\right)$ and $\gamma\left(W_{1}, W_{2}, Q\left(V, W_{3}\right)\right)$. Without loss of generality we can assume that the vector fields $W_{1}, W_{2}, W_{3}$ are linearly independent (over $\mathbb{C}$ ). Then we can find uniquely determined complex functions $f_{1}, f_{2}, f_{3}$ such that

$$
V=f_{1} W_{1}+f_{2} W_{2}+f_{3} W_{3} .
$$

Then we get

$$
\begin{aligned}
& \rho\left(W_{1}\right) \gamma\left(V, W_{2}, W_{3}\right)+\rho\left(W_{2}\right) \gamma\left(W_{1}, V, W_{3}\right)+\rho\left(W_{3}\right) \gamma\left(W_{1}, W_{2}, V\right) \\
&=f_{1} \rho\left(W_{1}\right) \gamma\left(W_{1}, W_{2}, W_{3}\right)+f_{2} \rho\left(W_{2}\right) \gamma\left(W_{1}, W_{2}, W_{3}\right) \\
&+f_{3} \rho\left(W_{3}\right) \gamma\left(W_{1}, W_{2}, W_{3}\right) \\
&= \rho\left(f_{1} W_{1}+f_{2} W_{2}+f_{3} W_{3}\right) \gamma\left(W_{1}, W_{2}, W_{3}\right)=\rho(V) \gamma\left(W_{1}, W_{2}, W_{3}\right) .
\end{aligned}
$$

Finally, we obtain

$$
\begin{aligned}
& \gamma\left(Q\left(V, W_{1}\right), W_{2}, W_{3}\right)+\gamma\left(W_{1}, Q\left(V, W_{2}\right), W_{3}\right)+\gamma\left(W_{1}, W_{2}, Q\left(V, W_{3}\right)\right) \\
& \quad=\rho(V) \gamma\left(W_{1}, W_{2}, W_{3}\right) .
\end{aligned}
$$

which proves $\nabla_{V} \gamma=0$.

Let us continue with the main stream of the proof. We now use the complex connection $\nabla$. For $X, Y \in T M$ we denote $\nabla_{X}^{0} Y=\operatorname{Re} \nabla_{X} Y$ and $\nabla_{X}^{1} Y=\operatorname{Im} \nabla_{X} Y$. This means that $\nabla_{X} Y=\nabla_{X}^{0} Y+i \nabla_{X}^{1} Y$. For a real function $f$ on $M$,

$$
\begin{gathered}
\nabla_{X}(f Y)=\nabla_{X}^{0}(f Y)+i \nabla_{X}^{1}(f X), \\
\nabla_{X}(f Y)=(X f) Y+f \nabla_{X} Y=\left[(X f) Y+f \nabla_{X}^{0} Y\right]+i f \nabla_{X}^{1} Y,
\end{gathered}
$$

which implies that

$$
\nabla_{X}^{0}(f Y)=(X f) Y+f \nabla_{X}^{0} Y, \quad \nabla_{X}^{1}(f Y)=f \nabla_{X}^{1} Y .
$$

This shows that $\nabla^{0}$ is a real connection while $\nabla^{1}$ is a real tensor field of type $(1,2)$. Also

$$
\begin{aligned}
0=\nabla_{X} Y-\nabla_{Y} X-[X, Y] & =\nabla_{X}^{0} Y+i \nabla_{X}^{1} Y-\nabla_{Y}^{0} X-i \nabla_{Y}^{1} X-[X, Y] \\
& =\left[\nabla_{X}^{0} Y-\nabla_{Y}^{0} X-[X, Y]\right]+i\left[\nabla_{X}^{1} Y-\nabla_{Y}^{1} X\right],
\end{aligned}
$$

which shows that

$$
\nabla_{X}^{0} Y-\nabla_{Y}^{0} X-[X, Y]=0, \quad \nabla_{X}^{1} Y-\nabla_{Y}^{1} X=0 .
$$

These equations show that the connection $\nabla^{0}$ is symmetric, and that the tensor $\nabla^{1}$ is also symmetric. Moreover,

$$
\begin{gathered}
\nabla_{X}(J Y)=\nabla_{X}^{0}(J Y)+i \nabla_{X}^{1}(J Y) \\
\nabla_{X}(J Y)=J \nabla_{X} Y=J \nabla_{X}^{0} Y+i J \nabla_{X}^{1} Y
\end{gathered}
$$


which gives

$$
\nabla_{X}^{0} J=0, \quad \nabla_{X}^{1}(J Y)=J \nabla_{X}^{1} Y .
$$

For the real vectors $X, Y_{1}, Y_{2}, Y_{3} \in T M$ we can compute

$$
\begin{aligned}
0= & \left(\nabla_{X} \gamma\right)\left(Y_{1}, Y_{2}, Y_{3}\right)=X\left(\gamma\left(Y_{1}, Y_{2}, Y_{3}\right)\right) \\
& -\gamma\left(\nabla_{X} Y_{1}, Y_{2}, Y_{3}\right)-\gamma\left(Y_{1}, \nabla_{X} Y_{2}, Y_{3}\right)-\gamma\left(Y_{1}, Y_{2}, \nabla_{X} Y_{3}\right) \\
= & X\left(\gamma\left(Y_{1}, Y_{2}, Y_{3}\right)\right)-\gamma\left(\nabla_{X}^{0} Y_{1}+i \nabla_{X}^{1} Y_{1}, Y_{2}, Y_{3}\right) \\
& -\gamma\left(Y_{1}, \nabla_{X}^{0} Y_{2}+i \nabla_{X}^{1} Y_{2}, Y_{3}\right)-\gamma\left(Y_{1}, Y_{2}, \nabla_{X}^{0} Y_{3}+i \nabla_{X}^{1} Y_{3}\right) \\
= & X\left(\gamma\left(Y_{1}, Y_{2}, Y_{3}\right)\right)-\gamma\left(\nabla_{X}^{0} Y_{1}, Y_{2}, Y_{3}\right)-\gamma\left(Y_{1}, \nabla_{X}^{0} Y_{2}, Y_{3}\right)-\gamma\left(Y_{1}, Y_{2}, \nabla_{X}^{0} Y_{3}\right) \\
& -i\left[\gamma\left(\nabla_{X}^{1} Y_{1}, Y_{2}, Y_{3}\right)+\gamma\left(Y_{1}, \nabla_{X}^{1} Y_{2}, Y_{3}\right)+\gamma\left(Y_{1}, Y_{2}, \nabla_{X}^{1} Y_{3}\right)\right] \\
= & {\left[X\left(\gamma_{0}\left(Y_{1}, Y_{2}, Y_{3}\right)\right)-\gamma_{0}\left(\nabla_{X}^{0} Y_{1}, Y_{2}, Y_{3}\right)-\gamma_{0}\left(Y_{1}, \nabla_{X}^{0} Y_{2}, Y_{3}\right)-\gamma_{0}\left(Y_{1}, Y_{2}, \nabla_{X}^{0} Y_{3}\right)\right.} \\
& \left.+\gamma_{1}\left(\nabla_{X}^{1} Y_{1}, Y_{2}, Y_{3}\right)+\gamma_{1}\left(Y_{1}, \nabla_{X}^{1} Y_{2}, Y_{3}\right)+\gamma_{1}\left(Y_{1}, Y_{2}, \nabla_{X}^{1} Y_{3}\right)\right] \\
& +i\left[X\left(\gamma_{1}\left(Y_{1}, Y_{2}, Y_{3}\right)\right)-\gamma_{1}\left(\nabla_{X}^{0} Y_{1}, Y_{2}, Y_{3}\right)-\gamma_{1}\left(Y_{1}, \nabla_{X}^{0} Y_{2}, Y_{3}\right)\right. \\
& -\gamma_{1}\left(Y_{1}, Y_{2}, \nabla_{X}^{0} Y_{3}\right)-\gamma_{0}\left(\nabla_{X}^{1} Y_{1}, Y_{2}, Y_{3}\right)-\gamma_{0}\left(Y_{1}, \nabla_{X}^{1} Y_{2}, Y_{3}\right) \\
& \left.-\gamma_{0}\left(Y_{1}, Y_{2}, \nabla_{X}^{1} Y_{3}\right)\right] .
\end{aligned}
$$

This shows that the real part is zero. The complex part then leads to the same identity, and thus it is also zero. Using the relations between $\gamma_{0}$ and $\gamma_{1}$ gives

$$
\begin{aligned}
0= & X\left(\gamma_{0}\left(Y_{1}, Y_{2}, Y_{3}\right)\right)-\gamma_{0}\left(\nabla_{X}^{0} Y_{1}, Y_{2}, Y_{3}\right)-\gamma_{0}\left(Y_{1}, \nabla_{X}^{0} Y_{2}, Y_{3}\right)-\gamma_{0}\left(Y_{1}, Y_{2}, \nabla_{X}^{0} Y_{3}\right) \\
& -\gamma_{0}\left(J \nabla_{X}^{1} Y_{1}, Y_{2}, Y_{3}\right)-\gamma_{0}\left(Y_{1}, J \nabla_{X}^{1} Y_{2}, Y_{3}\right)-\gamma_{0}\left(Y_{1}, Y_{2}, J \nabla_{X}^{1} Y_{3}\right) \\
= & X\left(\gamma_{0}\left(Y_{1}, Y_{2}, Y_{3}\right)\right)-\gamma_{0}\left(\nabla_{X}^{0} Y_{1}+J \nabla_{X}^{1} Y_{1}, Y_{2}, Y_{3}\right) \\
& -\gamma_{0}\left(Y_{1}, \nabla_{X}^{0} Y_{2}+J \nabla_{X}^{1} Y_{2}, Y_{3}\right)-\gamma_{0}\left(Y_{1}, Y_{2}, \nabla_{X}^{0} Y_{3}+J \nabla_{X}^{1} Y_{3}\right) .
\end{aligned}
$$

Now define

$$
\tilde{\nabla}_{X} Y=\nabla_{X}^{0} Y+J \nabla_{X}^{1} Y
$$

It is easy to verify that $\tilde{\nabla}$ is a real connection. Moreover, the previous equation shows that

$$
\tilde{\nabla} \gamma_{0}=0 \text {. }
$$

Furthermore, it is very easy to see that the connection $\tilde{\nabla}$ is symmetric.

The inverse implication can also be proved easily.

Let us use the standard definition of integrability of a $k$-form $\omega$ on $M$, that is, every $x \in M$ has a neighbourhood $N$ such that $\omega$ has the constant expression in $d x^{i}, x^{i}$ being suitable coordinate functions on $N$.

COROLLARY 14. Let $\omega$ be a real 3-form on a six-dimensional differentiable manifold $M$ satisfying $\Delta\left(\omega_{x}\right)=\{0\}$ for any $x \in M$. Let $J$ be an almost complex structure on $M$ such that for any vector fields $X_{1}, X_{2}, X_{3} \in \mathfrak{X}(M)$,

$$
\omega\left(J X_{1}, X_{2}, X_{3}\right)=\omega\left(X_{1}, J X_{2}, X_{3}\right)=\omega\left(X_{1}, X_{2}, J X_{3}\right) .
$$


Then $\omega$ is integrable if and only if there exists a symmetric connection $\nabla$ preserving $\omega$, that is, $\nabla \omega=0$.

PROOF. Let $\nabla$ be a symmetric connection such that $\nabla \omega=0$. According to the previous proposition, $d \omega=0$ and $J$ is integrable. Then we construct the complex form $\gamma$ on $T^{\mathbb{C}} M$ of type $(3,0)$ such that $\operatorname{Re} \gamma \mid T_{x} M=\omega$, for any $x \in M$ (point by point, according to Proposition 8). Moreover, if $\omega$ is closed then so is $\gamma$. That is, $\gamma=f \cdot d z^{1} \wedge d z^{2} \wedge d z^{3}$, where $z^{1}, z^{2}$, and $z^{3}$ are (complex) coordinate functions on $M, d z^{1}, d z^{2}, d z^{3}$ are a basis of $\Lambda^{1,0} M$ and $f$ is a function on $M$. Furthermore,

$$
0=d \gamma=\partial \gamma+\bar{\partial} \gamma=\partial f \cdot d z^{1} \wedge d z^{2} \wedge d z^{3}+\bar{\partial} f \cdot d z^{1} \wedge d z^{2} \wedge d z^{3}
$$

Evidently $\partial \gamma=0$, which means that $\bar{\partial} f=0$ and $f$ is holomorphic. Now we exploit a standard trick. There exists a holomorphic function $F\left(z^{1}, z^{2}, z^{3}\right)$ such that $\left(\partial F / \partial z^{1}\right)=f$. We introduce new complex coordinates $\tilde{z}^{1}=F\left(z^{1}, z^{2}, z^{3}\right)$, $\tilde{z}^{2}=z^{2}$, and $\tilde{z}^{3}=z^{3}$. Then $\gamma=f d z^{1} \wedge d z^{2} \wedge d z^{3}=d \tilde{z}^{1} \wedge d \tilde{z}^{2} \wedge d \tilde{z}^{3}$. Now write $\tilde{z}^{1}=x^{1}+i x^{4}, \tilde{z}^{2}=x^{2}+i x^{5}$, and $\tilde{z}^{3}=x^{3}+i x^{6}$ for real coordinate functions $x^{1}, x^{2}$, $x^{3}, x^{4}, x^{5}$, and $x^{6}$ on $M$. Then

$$
\begin{aligned}
\operatorname{Re} \gamma= & \operatorname{Re}\left(d\left(x^{1}+i x^{4}\right) \wedge d\left(x^{2}+i x^{5}\right) \wedge d\left(x^{3}+i x^{6}\right)\right) \\
= & d x^{1} \wedge d x^{2} \wedge d x^{3}-d x^{1} \wedge d x^{5} \wedge d x^{6}+d x^{2} \wedge d x^{4} \wedge d x^{6} \\
& -d x^{3} \wedge d x^{4} \wedge d x^{5}
\end{aligned}
$$

and $\omega=(\operatorname{Re} \gamma) \mid T M$ is an integrable on $M$.

Conversely, if $\omega$ is integrable, then for any $x \in M$ there is a basis $d x_{1}, \ldots, d x_{6}$ of $T^{*} N$ in some neighbourhood $N \subset M$ of $x$ such that $\omega$ has a constant expression in all $T_{x} M, x \in N$. Then the flat connection $\nabla$ given by the coordinate system $x_{1}, \ldots, x_{6}$ is symmetric and $\nabla \omega=0$ on $N$. We use a partition of unity, and extend $\nabla$ over the whole manifold $M$.

We can reformulate Theorem 13 as 'the Darboux theorem for complex type forms'.

COROLLARY 15. Let $\omega$ be a real 3-form on a six-dimensional differentiable manifold $M$ satisfying $\Delta\left(\omega_{x}\right)=\{0\}$ for any $x \in M$. Let $J$ be an almost complex structure on $M$ such that for any vector fields $X_{1}, X_{2}, X_{3} \in \mathfrak{X}(M)$,

$$
\omega\left(J X_{1}, X_{2}, X_{3}\right)=\omega\left(X_{1}, J X_{2}, X_{3}\right)=\omega\left(X_{1}, X_{2}, J X_{3}\right) .
$$

Then $\omega$ is integrable if and only if the following conditions are satisfied:

(i) $d \omega=0$;

(ii) the almost complex structure $J$ is integrable.

OBSERVATION 16. There is an interesting relation between structures given by a form of complex type on six-dimensional vector spaces and $G_{2}$-structures on seven-dimensional vector spaces $\left(G_{2}\right.$ being the exeptional Lie group, the group of 
automorphisms of the algebra of Cayley numbers and also the group of automorphism of the 3-form given below), that is, structures given by a form of the type

$$
\begin{aligned}
& \alpha_{1} \wedge \alpha_{2} \wedge \alpha_{3}+\alpha_{1} \wedge \alpha_{4} \wedge \alpha_{5}-\alpha_{1} \wedge \alpha_{6} \wedge \alpha_{7}+\alpha_{2} \wedge \alpha_{4} \wedge \alpha_{6}+\alpha_{2} \wedge \alpha_{5} \wedge \alpha_{7} \\
& \quad+\alpha_{3} \wedge \alpha_{4} \wedge \alpha_{7}-\alpha_{3} \wedge \alpha_{5} \wedge \alpha_{6}
\end{aligned}
$$

where $\alpha_{1}, \ldots, \alpha_{7}$ are the basis of the vector space $V$. If we restrict a form of this type to any six-dimensional subspace of $V$ we get a form of complex type. Thus, any $G_{2}$ structure on a seven-dimensional manifold gives a structure of complex type on any six-dimensional submanifold. Thus we get a vast variety of examples.

Many examples of $G_{2}$ structures are known and they have been well studied. See, for example, $[\mathrm{J}]$.

\section{References}

[F] T. Friedrich, 'Spin(9)-structures and connections with totally skew-symmetric torsion', J. Geom. Phys. 47(2) (2003), 197-206.

$[H] \quad$ N. Hitchin, 'The geometry of three-forms in six dimensions', J. Differential Geom. 55 (2000), 547-576.

[J] D. D. Joyce, Compact Manifolds with Special Holonomy, Oxford Mathematical Monographs (Oxford University Press, Oxford, 2000).

[V] J. Vanžura, 'One kind of multisymplectic structures on 6-manifolds steps in differential geometry', Proceedings of the Colloquium on Differential Geometry, Debrecen, Hungary, 25-30 July, 2000, pp. 375-391.

[W] F. Witt, 'Special metric structures and closed forms', PhD thesis, 2005, Oxford, arxiv:math.DG/0502443.

MARTIN PANÁK, Department of Algebra and Geometry, Masaryk University Brno, Janackovo nam. 2a, 60200 Brno, Czech Republic

e-mail: naca@math.muni.cz

JIŘÍ VANŽURA, Institute of Mathematics, AS CR, Zizkova 22, 61662 Brno,

Czech Republic

e-mail: vanzura@ipm.cz 